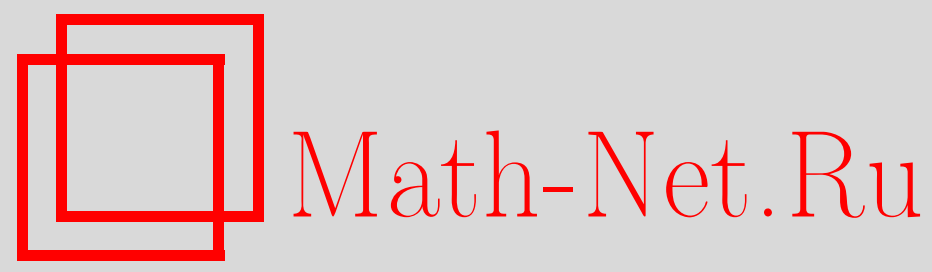

В. Д. Матвеенко, Оптимальные пути в ориентированных графах и собственные векторы в max- $\oplus$ системах, Дискрет. матем., 2009, том 21, выпуск 3, 79-98

DOI: https://doi.org/10.4213/dm1063

Использование Общероссийского математического портала Math-Net.Ru подразумевает, что вы прочитали и согласны с пользовательским соглашением http://www . mathnet.ru/rus/agreement

Параметры загрузки:

IP : 54.197 .217 .227

26 апреля 2023 г., $16: 31: 34$ 


\title{
Оптимальные пути в ориентированных графах и собственные векторы в $\max -\oplus$ системах
}

\author{
() 2009 г. $\quad$ В. Д. Матвеенко
}

\begin{abstract}
В задачах исследования операций и математической экономики возникают аналоги линейного оператора, где операции сложения и умножения чисел заменены соответственно на взятие максимума и некоторую бинарную операцию $\otimes$. Ранее изучались, в основном, два примера (и их обобщения), где в качестве $\otimes$ выступают сложение и минимум. В статье вводится в рассмотрение два других примера, где $\otimes-$ это сложение с дисконтированием (известное по экономической модели Рамсея-Касса-Купманса) и минимум с дисконтированием. Для исследования всех четырех примеров предлагается метод, в основе которого лежат операции над характеристическими парами путей в ориентированном графе. Характеристическая пара путей определена как пара чисел, одно из которых представляет собой вес пути (он определен посредством операции $\otimes$ ), а другой - число дуг пути. Основное внимание уделяется вычислению и свойствам собственного вектора оператора, он представляет собой функцию-значение Беллмана для соответствующей оптимизационной задачи о путях на графе.
\end{abstract}

\section{1. Введение}

В задачах исследования операций и математической экономики возникают аналоги линейного оператора, где операции сложения и умножения чисел заменены на некоторые другие бинарные операции $\oplus$ и $\otimes$ на числовом множестве $X$. Цель статьи - расширить класс имеющих прикладной интерес примеров и предложить методы исследования, применимые как к традиционным, так и к новым примерам.

Обычно в литературе рассматриваются следующие два примера, в которых $\oplus=\max$ (а также их аналоги с операцией min). Через 0 обозначается нулевой элемент относительно операции $\otimes$, то есть $g \otimes \mathbf{0}=\mathbf{0} \otimes g=\mathbf{0}$ для каждого $g \in X$.

Пример 1. Пусть $\otimes-$ сложение чисел, $X=\mathbf{R} \cup\{\infty\}, \mathbf{0}=-\infty$. (При мультипликативной записи $X=\mathbf{R}_{+}$, операция $\otimes$ представляет собой обычное умножение, $\left.\mathbf{0}=0\right)$. Этот пример, известный как схема динамического программирования, изучался, в частности, в [1-8]. В [5, 6] указаны приводящие к нему задачи исследования операций, такие, как выбор оптимального расписания работы станков и задача управления лесным хозяйством. Аналогичным образом записывается модель экономического развития [9].

Пример 2. Пусть $\otimes-$ операция взятия минимума. Известны два варианта этого примера (см. [10-13]). В так называемой нечеткой алгебре вектор $x \in X$ интерпретируется как вектор нечетких характеристик системы, и предполагается, что $X \in[0,1], \mathbf{0}=0$. Например, в [10] компоненты вектора $x$ представляют собой симптомы болезни, а матрица $A$, на 
которую умножается (в смысле операций $\otimes=\min , \oplus=\max )$ вектор $x$, отражает определенный метод лечения. В другом варианте теории, алгебре узких мест, $X=(-\infty,+\infty)$, элемент 0 отсутствует.

Конкретизация множества $X$ в примере 2 обычно оказывается несущественной: большинство результатов сохраняется. Мы при рассмотрении примера 2 будем считать, что $X=\mathbf{R}_{+}, \mathbf{0}=0$.

В этой статье наряду с примерами 1, 2 будем рассматривать еще два примера, в которых также $\oplus=\max$.

Пример 3. Операция $\otimes$ определяется следующим образом: $g \otimes h=g+\beta h$, где $g, h \in X$, $X=\mathbf{R} \cup\{-\infty\}, \beta \in(0,1)-$ заданное число (дисконтирующий множитель), $\mathbf{0}=-\infty$. Пример 3 соответствует схеме с дисконтированием [14], которая возникает в экономике (вариант модели Рамсея, см., напр., [15]), в финансовых расчетах и в исследовании операций (см., напр., гл. 11 в [16]).

Пример 4. Операция $\otimes$ определяется как $g \otimes h=\min \{g, \delta h\}$, где $X=\mathbf{R}_{+}, \delta>1-$ заданное число, $\mathbf{0}=0$. Пример 4 развивает пример 2 и может служить моделью ситуации, когда цель состоит в выборе траектории с максиминном доходом (то есть в том, чтобы избежать периода низких доходов), но при этом более отдаленный по времени будущий провал дохода воспринимается в момент принятия решения как менее существенный по сравнению с более близким по времени.

Элементы степенной матрицы $A^{\otimes t}$ (где степень определена в смысле операций max, $\otimes)$ имеют вполне определенный смысл, существенный для приложений. В примере 1 элемент $(i, j)$ степенной матрицы представляет собой значение задачи о построении в соответствующем матрице $A$ ориентированном графе $t$-шагового пути, ведущего из вершины $i$ в вершину $j$ и обладающего максимальным суммарным весом, в примере $2-$ значение задачи о максиминном пути, в примере 3 - о пути с максимальной суммарной дисконтированной полезностью, в примере 4 - о максиминном пути с дисконтированием.

Собственные векторы так же, как в обычной алгебре матриц, играют центральную роль для понимания структуры рекуррентных последовательностей, получаемых умножением матрицы на вектор, начиная с заданного начального вектора.

Рядом авторов предложены весьма общие математические конструкции, обобщающие примеры 1 и 2, они однако не могут быть непосредственно применены к примерам 3 и 4. Так, например, в $[17,18]$ рассматривается конструкция эндоморфизма полумодуля над полукольцом, обобщающая ситуацию примера 1. Однако наличие предположения о коммутативности операции $\otimes$ делает результаты работ $[17,18]$ неприменимыми к примерам 3,4 , а принятое там условие сокращения (из $a \otimes b=a \otimes c, a \neq \mathbf{0}$ следует равенство $b=c$ ) не соответствует примерам 2, 4. В [19], доказывая обобщенную теорему ПерронаФробениуса, авторы опираются на свойство однородности $(A \otimes(\lambda \otimes b)=\lambda \otimes(A \otimes b)$, где $A$ - матрица, $\lambda$ - число, $b-$ вектор, $\lambda \otimes b=\left(\lambda \otimes b_{1}, \lambda \otimes b_{2}, \ldots, \lambda \otimes b_{n}\right)^{\prime}$, штрих - знак транспонирования), которое не выполняется для примеров 3, 4. В [20] предполагается ассоциативность операции $\otimes$, которая также не имеет места в примерах 3,4 .

В настоящей статье предлагается подход, применимый для анализа всех четырех примеров. Основную роль играют понятия характеристической пары и произведения характеристических пар, они вводятся в разделе 2 . В разделе 3 перечислены основные свойства характеристических пар, которыми обладают все четыре примера и которые определяют структуру собственных векторов. В разделе 4 определено некоторое конечное множество бесконечных траекторий (класс $K$ ) и описано построение с его помощью собственного 
вектора. В разделе 5 получены условия единственности собственного вектора, доказано, что построенный собственный вектор является для примеров 3, 4 единственным, а для примера 2 максимальным. В разделе 6 с общих позиций обсуждаются известные алгоритмы вычисления максимального собственного вектора для примера 2 и предлагается алгоритм вычисления собственного вектора для примера 4. В разделе 7 для того же примера рассматривается задача построения множества всех собственных векторов. В разделе 8 дается определение левого собственного вектора, применимое для всех четырех примеров, и устанавливается роль левого собственного вектора в примере 3.

\section{2. Основные понятия}

Пусть задана матрица $A$ размера $n \times n$, элементы $a_{i j}$ которой принадлежат некоторому числовому множеству $X$, возможно, расширенному путем включения элемента $-\infty$. На множестве $X$ определена бинарная операция $\otimes$, причем существует нулевой элемент $\mathbf{0}$.

Пусть $a, b-n$-мерные векторы с элементами из $X$. Их произведением называется число

$$
a \otimes b=\max _{j=1, \ldots, n}\left\{a_{j} \otimes b_{j}\right\} .
$$

Заметим, что в примерах 3 и 4 произведение векторов (как и произведение чисел) не обладает ни коммутативностью, ни ассоциативностью.

Произведением матрицы $A$ на вектор $b$ называется $n$-мерный вектор $A \otimes b=c$ с элементами

$$
c_{i}=\max _{j=1, \ldots, n}\left\{a_{j} \otimes b_{j}\right\}, \quad i=1, \ldots, n .
$$

Пусть $B$ - матрица размера $n \times n$. Произведением матрицы $A$ на матрицу $B$ называется матрица $A \otimes B=C$ с элементами

$$
c_{i j}=\max _{k=1, \ldots, n}\left\{a_{i k} \otimes b_{k j}\right\}, \quad i, j=1, \ldots, n .
$$

Если $T-$ натуральное число, то $T$-й степенью матрицы $A$ называется матрица $A^{\otimes T}=A \otimes \ldots \otimes A$, элементы которой равны

$$
a_{i j}^{(T)}=\max _{i_{0}=i, i_{1}, \ldots, i_{T-1}, i_{T}=j} a_{i_{0} i_{1}} \otimes a_{i_{1} i_{2}} \otimes \ldots \otimes a_{i_{T-1} i_{T}}, \quad i, j=1, \ldots, n .
$$

При отсутствии ассоциативности, как обычно, операции выполняются, начиная справа.

Матрице $A$ соответствует ориентированный граф $G=\langle M, N\rangle$ с множествами вершин $M=\{1, \ldots, n\}$ и дуг $N=\left\{(i, j): i, j \in M, a_{i j} \neq \mathbf{0}\right\}$ с весами $a_{i j}$. Конечная последовательность $\left\{i_{0}, i_{1}, \ldots, i_{T}\right\}$ называется $T$-шаговой $\left(i_{0}, i_{T}\right)$-траекторией или $T$-звенным $\left(i_{0}, i_{T}\right)$-путем, если $\left(i_{k}, i_{k+1}\right) \in N, k=0,1, \ldots, T-1 . T$-шаговая траектория называется элементарной, если все ее вершины $i_{0}, i_{1}, \ldots, i_{T}$ различны. Аналогично, бесконечной траекторией с началом $i_{0}$ называется последовательность $\left\{i_{0}, i_{1}, \ldots\right\}$ такая, что $\left(i_{k}, i_{k+1}\right) \in N, k=0,1, \ldots$ Контуром называется $T$-шаговая траектория, начало и конец которой совпадают. Контур $\left\{i_{0}, i_{1}, \ldots, i_{T}=i_{0}\right\}$ называется элементарным, если его вершины $i_{0}, i_{1}, \ldots, i_{T-1}$ различны.

Будем предполагать, что граф $G$ является вполне связным, то есть для любой пары вершин $(i, j)$ существует $(i, j)$-путь. Как видно из (1), это предположение эквивалентно следующему: для каждой пары индексов $(i, j)$ существует такое натуральное число $T$, 
что элемент $(i, j)$ матрицы $A^{\otimes T}$ отличен от нулевого элемента, то есть $a_{i j}^{(T)} \neq \mathbf{0}$. Также эквивалентным является предположение о неразложимости матрицы $A$ : не существует такого подмножества индексов $S$, что $a_{i j}=\mathbf{0}$ при всех $i \notin S, j \in S$.

Весом $T$-звенного пути $s=\left\{i_{0}, i_{1}, \ldots, i_{T}\right\}$ будем называть величину

$$
c_{S}=a_{i_{0} i_{1}} \otimes \ldots \otimes a_{i_{T-1} i_{T}} .
$$

Через $v(i, j, T)$ обозначим значение задачи о нахождении максимального веса $T$-звенного $(i, j)$-пути. Как видно из $(1)$, элемент $(i, j)$ матрицы $A^{\otimes T}$ равен $v(i, j, T)$.

Собственным вектором матрицы $A$ будем называть вектор $x$, удовлетворяющий уравнению

$$
x=A \otimes x,
$$

то есть такой, что

$$
x_{i}=\max _{j=1, \ldots, n}\left\{a_{i j} \otimes x_{j}\right\}, \quad i=1,2, \ldots, n .
$$

В частности, в примере 3 это равенство принимает вид

$$
x_{i}=\max _{j=1, \ldots, n}\left\{a_{i j}+\beta x_{j}\right\}, \quad i=1,2, \ldots, n
$$

и представляет собой рекуррентное соотношение динамического программирования, определяющее функцию-значение Беллмана.

В литературе (см., напр., [20]) встречается и иное определение: вектор $y$ называется собственным вектором, а $\lambda$ - собственным числом матрицы $A$, если

$$
\lambda \otimes y=A \otimes y,
$$

где $\lambda \otimes y=\left(\lambda \otimes y_{1}, \ldots, \lambda \otimes y_{n}\right)^{\prime}$. Для примеров 2, 3, 4 естественным представляется определение (2). В примере 1 имеется простая связь между указанными двумя определениями. Матрица $A$ называется определенной [6], если ее собственное число равно 0. Посредством простого преобразования (вычитания собственного числа $\lambda$ из всех весов) произвольная матрица $A$ может быть сведена к определенной (см., напр., [4, 6, 7]), при этом равенство (3) заменяется на (2).

В примерах 1 и 2 собственный вектор матрицы $A$, вообще говоря, не единствен. Нахождению множества всех собственных векторов в примере 2 посвящен раздел 7.

Ниже $\oplus$ представляет собой максимум чисел или покоординатный максимум векторов.

В каждом из примеров 1-4 выполняется свойство дистрибутивности:

$$
g \otimes(h \otimes p)=(g \otimes h) \oplus(g \otimes p), \quad g, h, p \in X .
$$

Укажем некоторые свойства, которые вытекают из дистрибутивности.

Предложение 1. При наличии дистрибутивности, если $x, y-$ собственнье векторы матрицы A, то их сумма $x \oplus y$ также является собственным вектором.

Доказательство очевидно.

Упорядоченное множество, состоящее из $k$ различных векторов $x^{(1)}, \ldots, x^{(k)}$, назовем собственным циклом порядка $k$ матрицы $A$, если

$$
x^{(s+1)}=A \otimes x^{(s)}, \quad s=1, \ldots, k-1 ; \quad x^{(1)}=A \otimes x^{(k)} .
$$


Предложение 2. При наличии дистрибутивности, если векторы $x^{(1)}, \ldots, x^{(k)}$ составляют собственный ичикл порядка $k$ матрицы $A$, то их сумма $x^{(1)} \oplus \ldots \oplus x^{(k)}$ является собственным вектором.

Доказательство. Ясно, что

$$
\begin{aligned}
A \otimes\left(x^{(1)} \oplus \ldots \oplus x^{(k)}\right) & =\left(A \otimes x^{(1)}\right) \oplus \ldots \oplus\left(A \otimes x^{(k)}\right) \\
& =x^{(2)} \oplus \ldots \oplus x^{(k)} \oplus x^{(1)}=x^{(1)} \oplus \ldots \oplus x^{(k)} .
\end{aligned}
$$

Предложение доказано.

Предложение 3. При наличии дистрибутивности, если векторы $x^{(1)}, \ldots, x^{(k)}$ составляют собственный цุикл порядка $k$ матрицы $A$, то каждый из них является собственным вектором матриць $A^{\otimes k}$ и не является собственным вектором матрицьь $A^{\otimes l}$ ни для какого $l<k$.

Доказательство. Первое утверждение очевидно, докажем второе утверждение.

Если $x^{(i)}=A^{\otimes l} \otimes x^{(i)}, l<k$, то

$$
x^{(i)}= \begin{cases}x^{(i+l)}, & i \leqslant k-l, \\ x^{(i+l-k)}, & i>k-l,\end{cases}
$$

то есть не все векторы в наборе $x^{(1)} \ldots x^{(k)}$ различны, что противоречит определению собственного цикла порядка $k$. Предложение доказано.

Выполнения ассоциативности можно добиться, если перейти от множества $X$ к множеству $X \times \mathbf{N}$, где $\mathbf{N}-$ множество натуральных чисел.

Характеристической парой назовем $(a, k)$, где $a \in X, k \in \mathbf{N}$. В частности, характеристической парой $T$-звенного пути $s$ с весом $c_{s}$ назовем $\left(c_{s}, T\right)$.

Пусть определена операция умножения характеристической пары на число, которая ставит в соответствие характеристической паре $(a, k)$ и элементу $b \in X$ некоторый элемент $(a, k) \otimes b \in X$ так, что

$$
(a, 1) \otimes b=a \otimes b .
$$

Положим в примерах 1 и $2(a, k) \otimes b=a \otimes b$; в примере $3(a, k) \otimes b=a+\beta^{k} b$; в примере $4(a, k) \otimes b=\min \left\{a, \delta^{k} b\right\}$.

Определим произведение двух характеристических пар следующим образом:

$$
\left(a_{1}, k_{1}\right) \otimes\left(a_{2}, k_{2}\right)=\left(\left(a_{1}, k_{1}\right) \otimes a_{2}, k_{1}+k_{2}\right) .
$$

Видим, что характеристическая пара $T$-звенного пути $s=\left\{i_{0}, \ldots, i_{T}\right\}$ равна

$$
(c, T)=\left(a_{i_{0} i_{1}}, 1\right) \otimes \ldots \otimes\left(a_{i_{T-1} i_{T}}, 1\right) .
$$

Пусть $(a, k)$ - характеристическая пара. Ее $t$-й степенью, $t \in \mathbf{N}$, назовем характеристическую пару

$$
(a, k)^{\otimes t}=(a, k) \otimes \ldots \otimes(a, k) .
$$

Рассмотрим контур $\sigma=\left\{i_{1}, i_{2}, \ldots, i_{k}, i_{1}\right\}$ с числом дуг, равным $k$. Вершина $i_{1}$ фиксирована в качестве начальной. Вес (одного обхода) контура равен

$$
c_{\sigma}\left(i_{1}\right)=\left(a_{i_{1} i_{2}}, 1\right) \otimes \ldots \otimes\left(a_{i_{k-2} i_{k-1}}, 1\right) \otimes a_{i_{k-1} i_{k}} .
$$


Характеристической парой контура $\sigma$ является $\left(c_{\sigma}\left(i_{1}\right), k\right)$. Характеристическая пара пути, состоящего из $r$ полных обходов контура $\sigma$, равна $\left(c_{\sigma}\left(i_{1}\right), k\right)^{\otimes r}$. Весом бесконечного обхода контура $\sigma$ естественно назвать (в случае его существования в множестве $X$ ) предел

$$
v_{\sigma}\left(i_{1}\right)=\lim _{r \rightarrow \infty}\left(c_{\sigma}\left(i_{1}\right), k\right)^{\otimes r} \otimes c_{\sigma}\left(i_{1}\right) .
$$

\section{3. Свойства характеристических пар}

Перечислим некоторые свойства, которые выполняются в каждом из примеров 1-4. Мы увидим далее, что эти свойства гарантируют существование собственного вектора.

1. Монотонность. Пусть $a_{1}, a_{2}, b \in X, k \in \mathbf{N}$. Если $a_{1} \leqslant a_{2}$, то

$$
\left(a_{1}, k\right) \otimes b \leqslant\left(a_{2}, k\right) \otimes b, \quad(b, k) \otimes a_{1} \leqslant(b, k) \otimes a_{2} .
$$

В частности, $a_{1} \otimes b \leqslant a_{2} \otimes b, b \otimes a_{1} \leqslant b \otimes a_{2}$.

2. Невозрастание при умножении на нулевую характеристическую пару. Если $b \in X$, $b \geqslant 0, k \in \mathbf{N}$, то $(0, k) \otimes b \leqslant b$.

Из свойств 1,2 следует, что если $a, b \in X, a \leqslant 0 \leqslant b, k \in \mathbf{N}$, то

$$
(a, k) \otimes b \leqslant b .
$$

3. Однородность. Пусть $a, b, c \in X, k \in \mathbf{N}-$ натуральное число. Если $(a, k) \otimes b=c$, то $(\lambda a, k) \otimes \lambda b=\lambda c$ для любого числа $\lambda>0$.

Лемма 1. Пусть выполняются свойства монотонности и однородности, и числа $a_{1}, a_{2}, s \in X, k \in \mathbf{N}$ таковы, что $s a_{1}>0, a_{2} a_{1}>0, a_{1}=(s, k) \otimes a_{1}, a_{2}>(s, k) \otimes a_{2}$. Тогда $a_{1}<a_{2}$.

Доказательство. По свойству однородности

$$
a_{1} \frac{a_{2}}{a_{1}}=\left(s \frac{a_{2}}{a_{1}}, k\right) \otimes a_{1} \frac{a_{2}}{a_{1}},
$$

то есть

$$
a_{2}=\left(s \frac{a_{2}}{a_{1}}, k\right) \otimes a_{2} .
$$

Следовательно,

$$
\left(s \frac{a_{2}}{a_{1}}, k\right) \otimes a_{2}>(s, k) \otimes a_{2} .
$$

Отсюда с учетом монотонности следует, что $s \frac{a_{2}}{a_{1}}>s$, и значит, $a_{2}>a_{1}$. Лемма доказана.

4. Ассоциативность произведения характеристических пар. Пусть $a_{1}, a_{2}, a_{3} \in X$, $k_{1}, k_{2}, k_{3} \in \mathbf{N}$. Тогда

$$
\left(\left(a_{1}, k_{1}\right) \otimes\left(a_{2}, k_{2}\right)\right) \otimes\left(a_{3}, k_{3}\right)=\left(a_{1}, k_{1} \otimes\right)\left(\left(a_{2}, k_{2}\right) \otimes\left(a_{3}, k_{3}\right)\right) .
$$


5. Существование веса бесконечного обхода контура. В примере 1 с определенной матрицей вес бесконечного обхода контура $v_{\sigma}\left(i_{1}\right)$ всегда принимает одно из двух возможных значений 0 или $\mathbf{0}$. В примере 3

$$
v_{\sigma}\left(i_{1}\right)=\frac{c_{\sigma}\left(i_{1}\right)}{1-\beta^{k}} .
$$

В примерах 2 и 4

$$
v_{\sigma}\left(i_{1}\right)=c_{\sigma}\left(i_{1}\right)
$$

\section{4. Траектории класса $K$ и собственный вектор матрицы $A$}

Определим класс $K$ как множество траекторий, включающее

(1) бесконечные обходы элементарных контуров (с указанием вершины начала обхода) и

(2) траектории, состоящие из элементарного пути и бесконечного обхода элементарного контура (с указанием вершины начала обхода).

В случае 1 вес траектории равен весу бесконечного обхода контура. В случае 2 определим вес траектории как $\left(c_{s}, k_{s}\right) \otimes v_{\sigma}(i)$, где $c_{s}, k_{s}-$ соответственно, вес и число дуг элементарного пути $s, i$ - вершина, в которой заканчивается путь $s$ и с которой начинается обход элементарного контура $\sigma, v_{\sigma}(i)-$ вес бесконечного обхода контура $\sigma$.

Например, пусть в примере 1

$$
A=\left(\begin{array}{cc}
-\infty & 1 \\
-1 & -\infty
\end{array}\right) .
$$

Имеются две траектории класса $K$ с началом в вершине 1. Одна из них представляет собой бесконечный обход контура, начиная с вершины 1, ее вес равен 0. Другая траектория состоит из дуги $(1,2)$ и бесконечного обхода контура, начиная с вершины 2; вес этой траектории равен 1 . Имеются также две траектории класса $K$ с началом в вершине 2 , их веса равны 0 и -1 .

В классе $K$ конечное число траекторий. Следовательно, для каждой вершины $i \in M$ в этом классе существует траектория с началом $i$, обладающая максимальным весом $V(i)$; всякую такую траекторию будем называть оптимальной. Таким образом, определена функция $V(i), i \in M$.

Теорема 1. Вектор $V$ с координатами $V(i), i \in M$, является собственным вектором матрицы A.

Доказательство. Докажем, что для любой дуги $(i, j)$ графа $\langle M, N\rangle$ выполняется неравенство

$$
V(i) \geqslant a_{i j} \otimes V(j) .
$$

Предположим противное: существует дуга $(i, j)$, для которой

$$
V(i)<a_{i j} \otimes V(j) .
$$



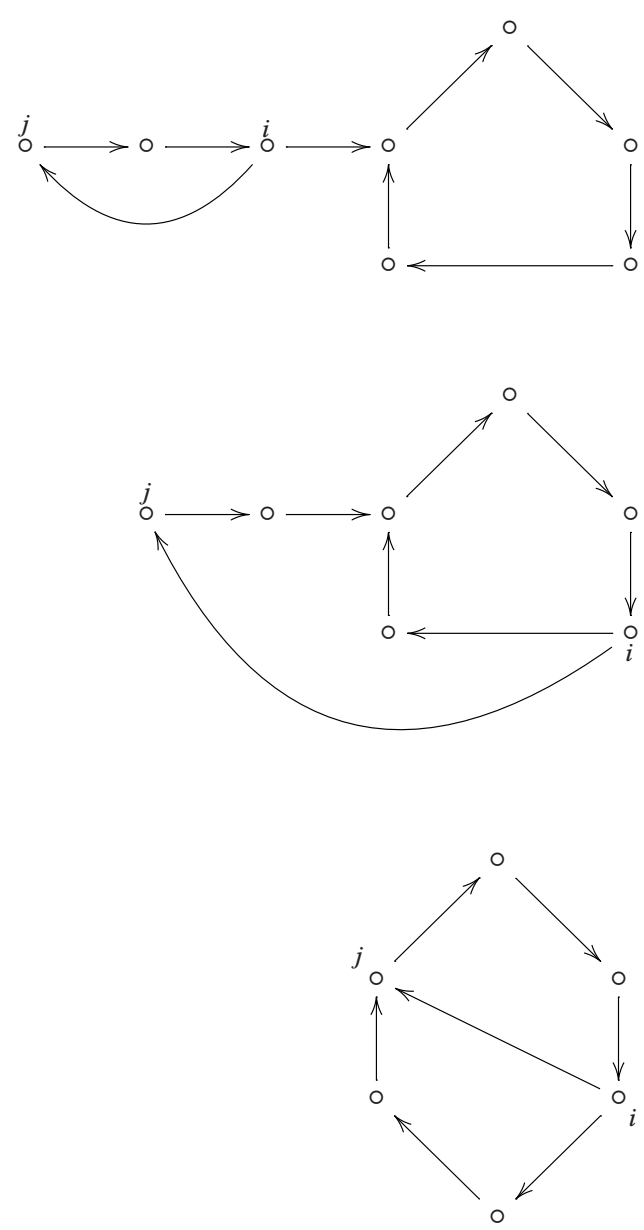

Рис. 1.

Пусть $\tau$ - оптимальная траектория класса $K$ с началом $j$, а $\tilde{\tau}$ - траектория, составленная из дуги $(i, j)$ и траектории $\tau$. Правая часть неравенства (6) представляет собой вес траектории $\tilde{\tau}$.

Если $\tau$ не проходит через вершину $i$, то $\tilde{\tau} \in K$, и неравенство (6) противоречит определению функции $V$.

Пусть теперь $\tau$ проходит через вершину $i$. Вершина $i$ может лежать на участке траектории $\tau$ до выхода на контур или на контуре $\sigma$. Возможные случаи показаны на рис. 1.

Обозначим через $\xi$ участок траектории $\tau$ от вершины $j$ до $i$, пусть он имеет вес $s(\xi)$ и число дуг $l(\xi)$. Этот участок не имеет самопересечений, поэтому контур, образованный добавлением дуги $(i, j)$, элементарен. Пусть $\bar{v}(i)-$ вес участка (хвоста) траектории $\tau$, начинающегося в вершине $i$. Тогда

$$
V(j)=(s(\xi), l(\xi)) \otimes \bar{v}(i)
$$


Поскольку $\bar{v}(i) \leqslant V(i)$, с учетом монотонности имеет место неравенство

$$
V(j) \leqslant(s(\xi), l(\xi)) \otimes V(i) .
$$

Отсюда и из (6) следует, что

$$
V(j)<\left(a_{i j}, 1\right) \otimes(s(\xi), l(\xi)) \otimes V(i) .
$$

Здесь $\left(a_{i j}, 1\right) \otimes(s(\xi), l(\xi))$ представляет собой характеристическую пару элементарного контура $\sigma$, образованного дугой $(i, j)$ и $(j, i)$-путем $\xi$. Пусть $v_{\sigma}(i)-$ вес бесконечного обхода контура $\sigma$, начиная с вершины $i$. Тогда

$$
v_{\sigma}(i)=\left(a_{i j}, 1\right) \otimes(s(\xi), l(\xi)) \otimes v_{\sigma}(i)
$$

Если $a_{i j} \otimes s(\xi)>0$, то, как следует из определения величин $V(i)$ и $v_{\sigma}(i)$, выполняется неравенство $V(i) \geqslant v_{\sigma}(i)>0$. Если $a_{i j} \otimes s(\xi) \leqslant 0$, то из (4) и (7) следует, что $V(i)<0$, кроме того, $v_{\sigma}(i)<0$. Равенство $a_{i j} \otimes s(\xi)=0$ невозможно (оно влечет неравенство $v_{\sigma}(i)=0>V(i)$, противоречащее определению функции $\left.V\right)$.

Таким образом, величины $a_{i j} \otimes s(\xi), v_{\sigma}(i), V(i)$ имеют одинаковый знак. По лемме 1 из (7) и (8) вытекает неравенство $V(i)<v_{\sigma}(i)$, что противоречит определению функции $V$.

Полученное противоречие означает справедливость неравенства (5).

Пусть теперь $(i, j)$ - дуга оптимальной траектории класса $K$ с началом в вершине $i$. На этой дуге $V(i)=a_{\overline{i j}} \otimes V(\bar{j})$. Следовательно,

$$
V(i)=\max _{j \in M}\left\{a_{i j} \otimes V(i)\right\}
$$

при всех $i \in M$. Теорема доказана.

\section{5. О единственности собственного вектора}

Как уже отмечалось, из определения функции $V$ следует, что для каждой вершины $i \in M$ и для каждого элементарного контура $\sigma$, содержащего вершину $i$, выполняется неравенство

$$
V(i) \geqslant v_{\sigma}(i) \text {. }
$$

Введем в рассмотрение условие

$$
\lim _{t \rightarrow \infty}\left(v_{\sigma}(i), t\right) \otimes V(i)=v_{\sigma}(i) .
$$

В примере 1 с определенной матрицей $A$, условие (10) справедливо, если единственным оптимальным контуром является петля. Этот случай часто встречается на практике. В примере 2 условия (9) и (10) эквивалентны. В примерах 3 и 4 равенство (10) выполняется автоматически.

Предложение 4. Возможен не более чем один собственный вектор, удовлетворяющий условию (10) для любого $i \in M$ и для любого элементарного контура $\sigma$, содержащего $i$.

Доказательство. Пусть $V_{i}$ - собственный вектор, обладающий указанным свойством. Для любой вершины $i$ можно указать траекторию $\tau$ класса $K$ с началом $i$, для каждой дуги которой $(\bar{i}, \bar{j})$ выполняется равенство

$$
V_{1}(\bar{i})=a_{\bar{i} \bar{j}} \otimes V_{1}(\bar{j}) .
$$


В общем случае, траектория $\tau$ включает элементарный путь $\xi$ из вершины $i$ в некоторую вершину $k$ и бесконечный обход некоторого контура $\sigma$, начиная с вершины $k$. Тогда, с учетом (10),

$$
V_{1}(i)=(s(\xi), l(\xi)) \otimes v_{\sigma}(k),
$$

то есть величина $V_{1}(i)$ равна весу траектории $\tau$ класса $K$ с началом $i$.

Рассмотрим теперь произвольную траекторию $\tau_{1}$ класса $K$ с тем же началом $i$, пусть она включает элементарный путь $\xi$ из вершины $i$ в вершину $k_{1}$ и обход контура $\sigma_{1}$, начинающийся с вершины $k_{1}$. Поскольку $V_{1}-$ собственный вектор, для каждой дуги $(\bar{i}, \bar{j}) \in N$ выполняется соотношение $V_{1}(\bar{i})=\left(a_{\bar{i}} \bar{j}, 1\right) \otimes V_{1}(\bar{j})$, и значит, с учетом условия $(10)$,

$$
V_{1}(i)=(s(\xi), l(\xi)) \otimes v_{\sigma_{1}}\left(k_{1}\right) .
$$

Следовательно, $V_{1}(i)$ представляет собой максимальный вес траектории класса $K$ с началом $i$, и значит, $V_{1}(i)=V(i), i \in M$. Предложение доказано.

Следствие 1. В примерах 3 и 4 собственный вектор является единственным.

Предложение 5. В примере 2 собственный вектор $V$ является максимальным, то есть для любого другого собственного вектора $\bar{V}$ выполняются неравенства $V(i) \geqslant \bar{V}(i)$, $i \in M$, причем хотя бы одно из этих неравенств строгое.

Доказательство. Для произвольной вершины $i$ рассмотрим траекторию $\tau$ класса $K$ с началом $i$ такую же, как в доказательстве предложения 4. Пусть траектория $\tau$ включает элементарный путь $\xi$ и обход контура $\sigma$, начинающийся с вершины $k$. Тогда

$$
\bar{V}(i)=s(\xi) \otimes \bar{V}(k), \quad \bar{V}(i)=c_{\sigma}(k) \otimes \bar{V}(k) .
$$

Поскольку $\otimes=\min$, отсюда следует, что $\bar{V}(i) \leqslant c_{\sigma}(k)=v_{\sigma}(k)$, и значит,

$$
\bar{V}(i)=s(\xi) \otimes v_{\sigma}(k) \leqslant V(i), \quad i \in M .
$$

С учетом эквивалентности условий (9) и (10) из предложения 4 следует существование вершины $i_{1}$ и контура $\sigma_{1}$ таких, что $\bar{V}\left(i_{1}\right)<v_{\sigma}\left(i_{1}\right) \leqslant V\left(i_{1}\right)$. Предложение доказано.

\section{6. Поведение рекуррентной последовательности $x^{(k+1)}=A \otimes x^{(k)}$ и построение}

\section{собственного вектора}

Рассмотрим последовательность векторов $\left\{x^{(k)}\right\}$, заданную рекуррентными соотношениями

$$
x^{(k+1)}=A \otimes x^{(k)}, \quad k=1,2, \ldots
$$

Можно убедиться, что в примере 4, независимо от начального вектора $x^{(1)}$, последовательность стабилизируется, совпадая с некоторого момента с собственным вектором матрицы. Как показано в [7, 8], в примерах 1 (с определенной матрицей) и 3 аналогичное свойство имеет место, если подматрица, соответствующая дугам оптимальных контуров, неразложима и примитивна.

Для примера 2 известно, что, хотя при произвольном начальном векторе $x^{(1)}$ последовательность $\left\{x^{(k)}\right\}$ не обязана сходиться (возможны циклы), сходимость к максимальному 
собственному вектору имеет место, если в качестве начального вектора $x^{(1)}$ взять вектор, составленный из максимальных элементов строк матрицы $A$,

$$
x_{i}^{(1)}=\max _{j=1, \ldots, n} a_{i j}, \quad i=1, \ldots, n \text {. }
$$

Этот факт связан со следующим свойством, которое справедливо для всех четырех примеров.

Предложение 6. Пусть последовательность $\left\{x^{(k)}\right\}$ определена рекуррентныли соотношениями (11), а начальный вектор $x^{(1)}$ составлен из максимальных элементов строк матриць А. Тогда каждый вектор $x^{(k)}, k=2,3, \ldots$, состоит из максимальных элементов строк матрицы $A^{\otimes k}$, то есть

$$
x_{i}^{(k)}=\max _{j=1, \ldots, n} a_{i j}^{(k)}, \quad i=1, \ldots, n .
$$

Доказательство. Нетрудно видеть, что

$$
\begin{aligned}
x_{i}^{(k)} & =\max _{j=1, \ldots, n}\left\{\left(a_{i j}^{(k-1)}, k-1\right) \otimes x_{j}^{(1)}\right\} \\
& =\max _{j=1, \ldots, n}\left\{\left(a_{i j}^{(k-1)}, k-1\right) \otimes \max _{s=1, \ldots, n} a_{j s}\right\} \\
& =\max _{s=1, \ldots, n} \max _{j=1, \ldots, n}\left\{\left(a_{i j}^{(k-1)}, k-1\right) \otimes a_{j s}\right\} \\
& =\max _{s=1, \ldots, n} a_{i s}^{(k)}, \quad i=1, \ldots, n, \quad k=2,3, \ldots
\end{aligned}
$$

Предложение доказано.

Для примера 2 свойства последовательности $\left\{x^{(k)}\right\}$ уточняются следующим образом.

Предложение 7. В примере 2 в условиях предложения 6 последовательность $\left\{x^{(k)}\right\}$ cmaбилизируется не позднее шага $n$, при этом $x^{(k)}=V, k=n, n+1, \ldots$, где $V-$ максимальный собственный вектор матрицыь $A$.

Доказательство. Пусть $i \in M$, и $\tau$ - траектория с началом $i$ (T-шаговая или бесконечная), имеющая в своем составе хотя бы один контур. Вводной частью траектории $\tau$ назовем ее участок, который начинается в вершине $i$ и заканчивается первой повторившейся вершиной. Заметим, что

- число дуг во вводной части не превышает $n$;

- всякая $T$-шаговая траектория при $T>n$ имеет вводную часть;

- если $v(\tau)$ - вес траектории $\tau, v_{i n}(\tau)$ - вес ее вводной части, $\bar{v}(\tau)-$ вес (бесконечной) траектории класса $K$ с той же вводной частью, то

$$
\nu(\tau) \leqslant v_{\text {in }}(\tau)=\bar{v}(\tau) \leqslant V(i)
$$

- вес всякой траектории класса $K$ совпадает с весом любого ее начального участка с числом дуг $k, k \geqslant n$. 
Таким образом, величина $V(i)$ представляет собой максимум весов $k$-шаговых траекторий, $k \geqslant n$, с началом $i$, то есть

$$
V(i)=\max _{k=n, n+1, \ldots} \max _{s=1, \ldots, n} a_{i s}^{(k)},
$$

и поэтому $x^{(k)}=V, k=n, n+1 \ldots$, в силу предложения 6. Предложение доказано.

Введем в рассмотрение множества

$$
I_{\min }\left(x^{(k)}\right)=\operatorname{Arg} \min _{i=1, \ldots, n} x_{i}^{(k)}=\left\{s: x_{s}^{(k)}=\min _{i=1, \ldots, n} x_{i}^{(k)}\right\} .
$$

Предложение 8. В примерах 2 и 4 в условиях предложения 6 , если $\bar{i} \in I_{\min }\left(x^{(1)}\right)$, то есть, если

$$
x_{\bar{i}}^{(1)}=a=\min _{i=1, \ldots, n} \max _{j=1, \ldots, n} a_{i j},
$$

mo

$$
\bar{i} \in I_{\min }\left(x^{(k)}\right), \quad x_{\bar{i}}^{(k)}=a, \quad k=2,3, \ldots, n+1 .
$$

Доказательство. Покажем, что при всех $k=1,2, \ldots$ выполняются неравенства $x_{i}^{(k)} \geqslant a$, $i=1, \ldots, n$, причем при $i \in I_{\min }\left(x^{(1)}\right)$ имеют место равенства.

Базу индукции получаем при $k=1$. Покажем, что если указанные свойства выполняются при некотором $k=s$, то они выполняются и при $k=s+1$. Действительно,

$$
x_{i}^{(s+1)}=\max _{j=1, \ldots, n}\left(a_{i j} \otimes x_{j}^{(s)}\right) \geqslant \max _{j=1, \ldots, n}\left(a_{i j} \otimes a\right)=\left(\max _{j=1, \ldots, n} a_{i j}\right) \otimes a=a, \quad i=1, \ldots, n .
$$

Если $\bar{i} \in I_{\min }\left(x^{(1)}\right)$, то $\max _{j=1, \ldots, n} a_{i j}=a$, и значит

$$
x_{\bar{i}}^{(s+1)}=\max _{j=1, \ldots, n}\left(a_{\bar{i} j} \otimes x_{j}^{(s)}\right) \leqslant a \otimes \max _{j=1, \ldots, n} x_{j}^{(s)}=a,
$$

следовательно,

$$
x_{\bar{i}}^{(s+1)}=a=\min _{i=1, \ldots, n} x_{i}^{(s+1)} .
$$

Предложение доказано.

Для примера 2 в $[10,12,13]$ предложен алгоритм вычисления максимального собственного вектора, менее трудоемкий по сравнению с непосредственным применением рекуррентного соотношения (11). Этот алгоритм состоит из следующих шагов.

Шаг 1. Положим $K=\{1, \ldots, n\}, \tilde{b}=0$.

Шаг 2. Вычислим значения $b_{i}=\max _{j \in K} a_{i j}$ при $i \in K$; вычислим $b=\min _{i \in K} b_{i}$ и положим $\tilde{I}=\left\{i: b_{i}=b\right\}$.

Шаг 3. Если $b>\tilde{b}$, то положим $\tilde{b}=b$.

Шаг 4. Положим $x_{i}^{*}=\tilde{b}$ при $i \in \tilde{I}$.

Шаг 5. Исключим из множества $K$ индексы, входящие в множество $\tilde{I}$.

Шаг 6. Если $K=\varnothing$, то вычисление максимального собственного вектора $x^{*}$ закончено. В противном случае перейдем к шагу 2. 
На каждой итерации вычисляются оптимальные значения одного или нескольких элементов максимального собственного вектора. Обоснование этого алгоритма легко проводится на основе предложений 6-8.

Для вычисления собственного вектора в примере 4 можно предложить следующий алгоритм того же типа.

Шаг 1. Положим $K=\{1, \ldots, n\}, L=\varnothing$.

Шаг 2. Вычислим значения $b_{i}=\max _{j \in K \cup L} a_{i j}$ при $i \in K$; вычислим $b=\min _{i \in K} b_{i}$, и положим $\tilde{I}=\left\{i: b_{i}=b\right\}$.

Шаг 3. Положим $x_{i}^{*}=b$ при $i \in \tilde{I}$.

Шаг 4. Исключим из множества $K$ индексы, входящие в множество $\tilde{I}$.

Шаг 5. Если $K=\varnothing$, то вычисление максимального собственного вектора $x^{*}$ закончено. В противном случае перейдем к шагу 6.

Шаг 6. Вычислим значения

$$
c_{i}=\min \left\{\max _{j \in \tilde{I}} a_{i j}, \delta b\right\}, \quad i \in K .
$$

Шаг 7. Включим в множество $L$ новый индекс $l$. Положим $a_{i l}=c_{i}, i \in K$. Перейдем к шагу 2.

\section{7. Другие собственные векторы в примере 2}

В примере 2, помимо максимального собственного вектора, представляет интерес множество всех собственных векторов. Например, в медицинской интерпретации (см. раздел 1) максимальный собственный вектор описывает наихудшее состояние больного, которое остается стабильным при данном методе лечения, а другие собственные векторы другие стабильные состояния.

Лемма 2. В примере 2 вектор х является собственным вектором матрицы $A$ тогда $u$ только тогда, когда

(1) для любой вершины $i, i=1, \ldots, n$, в графе $G$ существует дуга $(i, j)$ такая, что $a_{i j} \geqslant x_{i}, x_{j} \geqslant x_{i}, u$

(2) не существует дуги $(i, j)$ такой, что $a_{i j}>x_{i}, x_{j}>x_{i}$.

Утверждение непосредственно следует из определения собственного вектора.

Следствие 2. Если $x$ - собственный вектор матрицьы A, то

$$
x_{i} \leqslant \max _{j=1, \ldots, n} a_{i j}, \quad i=1, \ldots, n \text {. }
$$

Доказательство. Предположим противное, пусть $x_{\bar{i}}>\max _{j=1, \ldots, n} a_{\bar{i} j}$ для некоторого индекса $\bar{i}$. Тогда нарушено первое условие леммы 2 , и в силу этой леммы вектор $x$ не является собственным, что противоречит условию.

Следующие три следствия были доказаны в [12] как самостоятельные теоремы. 
Следствие 3. Произвольный вектор $x=(\mu, \ldots, \mu)$ (тривиальный в терминологии [12]) при $0 \leqslant \mu \leqslant \min _{i=1, \ldots, n} \max _{j=1, \ldots, n} a_{i j}$ является собственным вектором матрицьы $A$.

Доказательство. Поскольку $\max _{j=1, \ldots, n} a_{i j}>\mu$ для любой вершины $i$, выполняется первое условие леммы 2. Второе условие леммы выполнено тривиально. В силу этой леммы, $x$ является собственным вектором.

Следствие 4. При $\mu>\min _{i=1, \ldots, n} \max _{j=1, \ldots, n} a_{i j}$ ни один вектор $x=(\mu, \ldots, \mu)$ не является собственным вектором матрицы $A$.

Утверждение непосредственно вытекает из следствия 2.

Пусть фиксировано некоторое вещественное число $\mu$. Пороговым графом $G(\mu)$ называется подграф с множеством дуг, состоящим из всех дуг множества $N$ с весами $a_{i j} \geqslant \mu$.

Вершина $i$ в некотором графе называется предциклической, если в данном графе существует путь с началом $i$, ведущий в какой-либо контур.

Следствие 5. Если $x$ - собственный вектор матрицы $A$, то каждая вершина $i=1, \ldots, n$ является предииклической в пороговом графе $G\left(x_{i}\right)$.

Доказательство. В силу леммы 2 выполняется ее первое условие, откуда следует существование траектории класса $K$ с началом $i$, последовательность весов дуг которой не убывает, и при этом все веса не меньше $x_{i}$. Таким образом, вся траектория лежит в графе $G\left(x_{i}\right)$.

Теорема 2. В примере 2 вектор х является собственным вектором матрииь А тогда и только тогда, когда выполняется следующее свойство: неравенство $x_{i} \geqslant \mu$ справедливо для тех и только тех индексов $i$, для которых в пороговом графе $G(\mu)$ имеется путь, ведущий из вершины $i$ в вершину $j$ такую, что $x_{j} \geqslant \mu u j$ принадлежит некоторому контуру графа $G(\mu)$.

Доказательство. Пусть $x$ - собственный вектор. Докажем необходимость в условии теоремы. Предположим, что $x_{i} \geqslant \mu$ для некоторого индекса $i$ и числа $\mu$. Тогда в силу первого условия леммы 2 существует траектория $\left\{i_{k}\right\}$ класса $K$ с началом $i$, на которой $x_{i_{k}} \geqslant \mu, a_{i_{k} i_{k+1}} \geqslant \mu, k=0,1, \ldots$

Докажем достаточность условий теоремы. Предположим противное, пусть $x_{i}<\mu$. Тогда в графе $G(\mu)$ на $(i, j)$-пути можно указать дугу $\left(i_{k}, i_{k+1}\right)$ такую, что $x_{i_{k}}<\mu$, $x_{i_{k+1}} \geqslant \mu, a_{i_{k} i_{k+1}} \geqslant \mu$, то есть $a_{i_{k} i_{k+1}}>x_{i_{k}}, x_{i_{k+1}}>x_{i_{k}}$. Это противоречит второму условию леммы 2 , то есть сделанное предположение неверно, значит, $x_{i} \geqslant \mu$.

Пусть выполняется условие теоремы. Проверим условия леммы 2.

Докажем первое условие. Пусть $i-$ некоторый индекс. Положим $\mu=x_{i}$. В силу условия теоремы в графе $G(\mu)$ имеется $(i, j)$-путь, для всех дуг которого $a_{i_{k} i_{k+1}} \geqslant \mu$, $x_{i_{k}} \geqslant \mu$. Следовательно, выполняется первое условие леммы 2.

Докажем второе условие леммы 2. Предположим противное, пусть в графе $G$ существует дуга $(i, j)$, для которой $a_{i j}>x_{i}, x_{j}>x_{i}$. Выберем $\mu$ так, что

$$
x_{i}<\mu<\min \left\{a_{i j}, x_{j}\right\} .
$$

Тогда в силу условия теоремы в графе $G(\mu)$ существует путь $\tau$, ведущий из вершины $j$ в такой контур $\sigma$, что $x_{k} \geqslant \mu$ для некоторой вершины $k \in \sigma$. Поскольку $a_{i j}>\mu$, в графе $G(\mu)$ имеется также $(i, k)$-путь. Следовательно, в силу достаточности в условии теоремы выполняется неравенство $x_{i} \geqslant \mu$, противоречащее (12), что означает справедливость второго условия леммы 2 . 
В силу леммы 2 вектор $x$ является собственным вектором матрицы $A$. Теорема доказана.

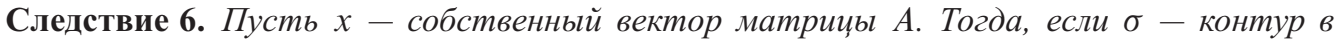
пороговом графе $G(\mu)$, то либо $x_{i} \geqslant \mu$ для всех $i \in \sigma$, либо $x_{i}<\mu$ для всех $i \in \sigma$.

Доказательство. Возможны два случая: либо $x_{i}<\mu$ для всех $i \in \sigma$, либо существует вершина $j \in \sigma$, для которой $x_{j} \geqslant \mu$. В последнем случае всякая вершина $i \in \sigma$ обладает свойством, составляющим правую часть свойства в теореме 2 . Согласно теореме $2, x_{i} \geqslant \mu$. Следствие доказано.

Теорема 2 и следствие 6 могут быть непосредственно использованы для построения множества всех собственных векторов матрицы $A$.

Пусть $\bar{\mu}$ - максимальное значение параметра $\mu$, при котором в пороговом графе $G(\mu)$ существует контур. Будем рассматривать пороговые графы $G(\mu)$, постепенно уменьшая значение параметра $\mu$, начиная с $\bar{\mu}$. При этом некоторым элементам $x_{i}$ собственного вектора (каким именно, уточняется ниже) будет присваиваться значение $\mu$. В каждый текущий момент будем называть помеченными вершины $i$, для которых уже определены значения $x_{i}$, а остальные вершины будем называть непомеченными.

Значения $x_{i}$ устанавливаются по следующим правилам, которые вытекают из теоремы 2 и следствия 6.

(1) Если при текущем значении $\mu$ в графе $G(\mu)$ имеется дуга $(i, j)$, ведущая из непомеченной вершины $i$ в помеченную вершину $j$, то должно быть присвоено значение $x_{i}=\mu$.

В частности, если в графе $G(\mu)$ имеется контур $\sigma$, некоторые, но не все вершины которого помечены, то все непомеченные вершины $i \in \sigma$ должны быть помечены с присвоением значения $x_{i}=\mu$.

(2) Если при текущем значении $\mu$ в графе $G(\mu)$ имеется контур $\sigma$, ни одна из вершин которого не помечена, то могут быть присвоены (или нет) значения $x_{i}=\mu$ для всех вершин $i \in \sigma$ одновременно.

(3) Переход к меньшему значению параметра $\mu$ производится, когда в графе $G(\mu)$ закончен перебор контуров с непомеченными вершинами, и не осталось ни одной дуги, ведущей из непомеченной вершины в помеченную.

Из теоремы 2 и следствия 6 вытекает, что каждый построенный вектор $x$ является собственным вектором матрицы $A$ и каждый собственный вектор может быть построен таким образом.

Пример 5. Пусть

$$
A=\left(\begin{array}{cc}
2 & 1 \\
2 & 1,5
\end{array}\right)
$$

тогда $\bar{\mu}=2$. Пороговый граф $G(2)$, как и все графы $G(\mu), 0 \leqslant \mu \leqslant 2$, содержит петлю $(1,1)$. При каждом $\mu$, если вершина 1 не помечена, может быть присвоено значение $x_{1}=\mu$, после чего в графе $G(\mu)$ имеется дуга $(2,1)$, ведущая из непомеченной вершины в помеченную, и должно быть присвоено значение $x_{2}=\mu$. Таким образом, в множестве собственных векторов имеется семейство $x=(\mu, \mu), 0 \leqslant \mu \leqslant 2$. 


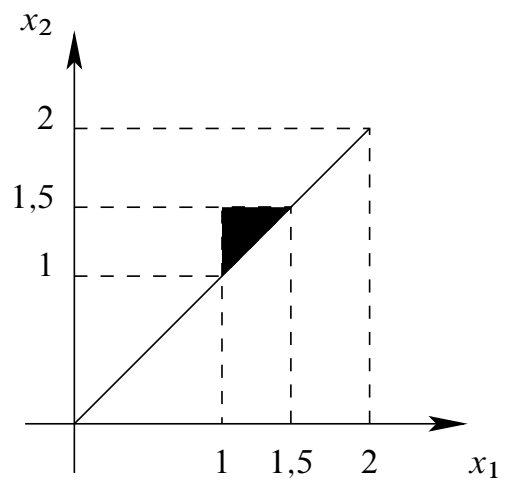

Рис. 2.

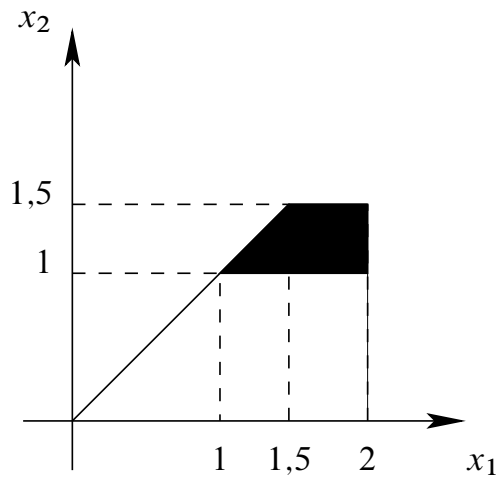

Pис. 3.

При $1 \leqslant \mu \leqslant 1,5$ граф $G(\mu)$ содержит петлю $(2,2)$, поэтому, если вершина 2 не помечена, может быть присвоено значение $x_{2}=\mu$. Вершину 1 при этом оставляем непомеченной, иначе будет получен собственный вектор из уже найденного выше семейства. Зафиксировав значение $x_{2}=\mu$ (пометив вершину 2), изменяем другой параметр $v$. При $1 \leqslant v \leqslant \mu$ может быть присвоено значение $x_{1}=v$. В случае же, когда вершина $i=1$, по мере уменьшения $v$, все же осталась непомеченной, она должна быть помечена при $v=1$, поскольку в графе $G(1)$ имеется дуга $(1,2)$, ведущая из непомеченной вершины в помеченную. Таким образом, построено еще одно семейство собственных векторов $x=(v, \mu)$, $1 \leqslant v<\mu \leqslant 1,5$. Множество всех собственных векторов матрицы $A$ изображено на рис. 2 .

Пример 6. Множество собственных векторов матрицы

$$
A=\left(\begin{array}{cc}
2 & 2 \\
1 & 1,5
\end{array}\right)
$$

изображено на рис. 3. 


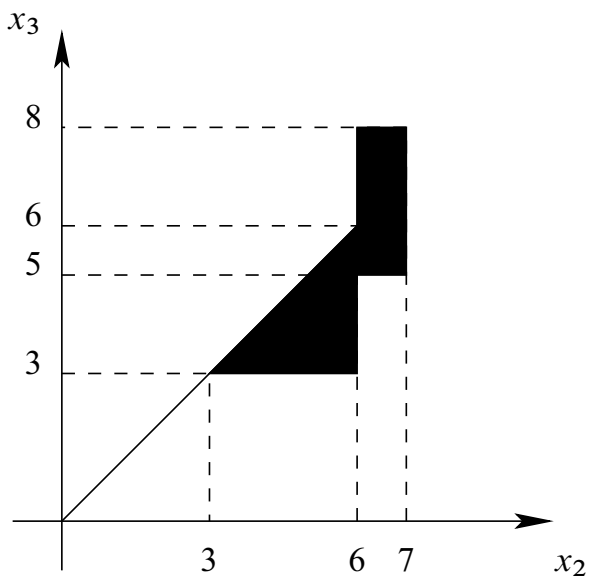

Pис. 4.

Пример 7. Множество собственных векторов матрицы

$$
A=\left(\begin{array}{lllll}
1 & 2 & 4 & 9 & 5 \\
1 & 1 & 2 & 6 & 7 \\
3 & 0 & 8 & 5 & 3 \\
1 & 2 & 6 & 7 & 1 \\
2 & 6 & 3 & 8 & 0
\end{array}\right)
$$

состоит из следующих семейств:

$$
\begin{aligned}
& \left(\begin{array}{lllll}
b & b & a & b & b
\end{array}\right), \quad 5 \leqslant b \leqslant a \leqslant 7 \text {; } \\
& \left(\begin{array}{lllll}
a & a & b & a & a
\end{array}\right), \quad a \in[6,7], b \in(6,8], a \leqslant b \text {; } \\
& \left(\begin{array}{lllll}
a & a & a & a & a
\end{array}\right), \quad a \in[0,7] \text {; } \\
& \left(\begin{array}{lllll}
5 & b & a & a & b
\end{array}\right), \quad a \in[3,5), b \in(5,6] \text {; } \\
& \left(\begin{array}{lllll}
b & b & a & a & b
\end{array}\right), \quad 3 \leqslant a \leqslant b \leqslant 5 \text {; } \\
& \left(\begin{array}{lllll}
b & c & a & b & c
\end{array}\right), \quad 5<a \leqslant b \leqslant c \leqslant 6 \text {. }
\end{aligned}
$$

На рис. 4 изображена проекция этого множества на плоскость $\left(x_{2}, x_{3}\right)$.

Помимо собственных векторов данная матрица $A$ обладает также собственными циклами порядка 2, то есть имеются пары векторов $x^{1}, x^{2}$ такие, что $A \otimes x^{1}=x^{2}, A \otimes x^{2}=x^{1}$. Замечая, что каждый из таких векторов является собственным вектором матрицы $A^{\otimes 2}$, находим следующие семейства собственных циклов:

$$
\begin{aligned}
& x^{1}=\left(\begin{array}{lllll}
a & b & a & a & c
\end{array}\right), \quad x^{2}=\left(\begin{array}{lllll}
a & c & a & a & b
\end{array}\right), \quad 5 \leqslant a<b<c \leqslant 6 ; \\
& x^{1}=\left(\begin{array}{lllll}
b & b & a & b & c
\end{array}\right), \quad x^{2}=\left(\begin{array}{lllll}
b & c & a & b & b
\end{array}\right), \quad 5<c<b<a \leqslant 6 ; \\
& x^{1}=\left(\begin{array}{lllll}
5 & c & a & a & b
\end{array}\right), \quad x^{2}=\left(\begin{array}{lllll}
b & b & a & a & c
\end{array}\right), \quad 3<a \leqslant b<5<c \leqslant 6 \text {; } \\
& x^{1}=\left(\begin{array}{lllll}
c & c & a & a & b
\end{array}\right), \quad x^{2}=\left(\begin{array}{lllll}
b & b & a & a & c
\end{array}\right), \quad 3 \leqslant a \leqslant b \leqslant 5, \quad c \in(b, 6) ; \\
& x^{1}=\left(\begin{array}{lllll}
b & d & a & b & c
\end{array}\right), \quad x^{2}=\left(\begin{array}{lllll}
b & c & a & b & d
\end{array}\right), \quad 5<a<b<c<d<6 .
\end{aligned}
$$




\section{8. Левые собственные векторы}

Левые собственные векторы наряду с правыми определяют структуру $T$-шаговых оптимальных траекторий (см. [7], где исследуется пример 1). В примерах 1, 2 и 4 естественно определить левый собственный вектор симметрично правому собственному вектору. Иначе обстоит дело в примере 3 , там, как мы сейчас увидим, при естественном определении левого собственного вектора левое собственное число не равно правому.

Определение левого собственного вектора, пригодное для всех четырех примеров, сформулируем по аналогии с очевидным свойством левого собственного вектора в обычной алгебре: если $p$ - левый собственный вектор матрицы $A$, соответствующий собственному числу $\lambda$, то $\lambda^{k} p A=\lambda^{k+1} p$ при всех $k=0,1, \ldots$

Если $b$ - вектор (строка), $A$ - матрица, через $(b, k) \otimes A$ обозначим вектор с элементами,

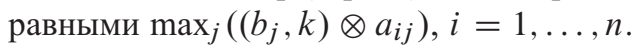

Вектор $p$ назовем левым собственным вектором (в смысле операций max, $\otimes$ ) матрицы $A$, а число $\mu$ - левым собственным числом, если

$$
\left(\mu^{k} p, k+1\right) \otimes A=\mu^{k+1} p, \quad k=0,1, \ldots
$$

Предложение 9. В примере 3 левое собственное число равно коэффициенту дисконтирования $\beta$.

Доказательство. По определению,

$$
\max _{i=1, \ldots, n}\left\{\mu^{k} p_{i}+\beta^{k+1} a_{i j}\right\}=\mu^{k+1} p_{j}, \quad j=1, \ldots, n,
$$

следовательно,

$$
\max _{i=1, \ldots, n}\left\{p_{i} / \mu+(\beta / \mu)^{k+1} a_{i j}\right\}=p_{j}, \quad j=1, \ldots, n
$$

при всех $k=0,1, \ldots$

Если $\mu \neq \beta$, то при изменении $k$ левая часть равенства изменяется (хотя бы для одного $j$, за исключением случая, когда $A=0$ ), тогда как правая часть постоянна. Следовательно, $\mu=\beta$. Предложение доказано.

Следствие 7. В примере 3 левый собственный вектор является решением уравнения $p \otimes A=\beta p$, то есть

$$
\max _{i=1, \ldots, n}\left\{p_{i}+\beta a_{i j}\right\}=\beta p_{j}, \quad j=1, \ldots, n .
$$

Таким образом, в примере 3 левый собственный вектор представляет собой вторую функцию-значение (см. [14, 15]).

Предложение 10. В примере 3, если $p$ - левый собственный вектор, a $\bar{x}$ - правый собственный вектор, то

$$
p \otimes \bar{x}=0
$$

Доказательство. Заметим, что для любых $n$-мерных векторов $s$ (строки) и $x$ (столбца)

$$
(s \otimes A, 2) \otimes x=s \otimes(A \otimes x) .
$$


Действительно, левая часть равна $\max _{i=1, \ldots, n}\left\{\max _{j=1, \ldots, n}\left\{s_{i}+\beta a_{i j}\right\}+\beta^{2} x_{j}\right\}$, а правая часть есть $\max _{i=1, \ldots, n}\left\{s_{i}+\beta \max _{j=1, \ldots, n}\left\{a_{i j}+\beta x_{j}\right\}\right\}$. Эти величины совпадают.

Возьмем в качестве $s$ левый собственный вектор $p$, а в качестве $x$ - правый собственный вектор $\bar{x}$. Тогда левая часть (14) с учетом следствия 7 равна $(\beta p, 2) \otimes \bar{x}=\beta(p \otimes \bar{x})$, а правая часть равна $p \otimes \bar{x}$. Отсюда вытекает (13). Предложение доказано.

Исследуем поведение последовательностей $\left\{x^{(k)}\right\}$ и $\left\{p^{(k)}\right\}$, определенных рекуррентными соотношениями

$$
x^{(k+1)}=A \otimes x^{(k)}, \quad p^{(k+1)}=p^{(k)} \otimes A, \quad k=0,1, \ldots
$$

Вспомним (см., напр., $[21,22])$, что в обычной алгебре, если $A-$ неразложимая примитивная матрица с неотрицательными элементами, $\alpha, \bar{x}, p-$ соответственно ее фробениусово собственное число, правый и левый фробениусовы собственные векторы такие, что

$$
p \bar{x}=1,
$$

To

$$
\lim _{k \rightarrow \infty} \alpha^{-k} A^{k}=\bar{A}
$$

где

$$
\bar{A}=\bar{x} p,
$$

то есть элементы матрицы $\bar{A}$ равны $\bar{a}_{i j}=\bar{x}_{i} p_{j}$. Если последовательности $\left\{x^{(k)}\right\},\left\{p^{(k)}\right\}$ определены равенствами

$$
x^{(k+1)}=A x^{(k)}, \quad p^{(k+1)}=p^{(k)} A, \quad k=0,1, \ldots,
$$

To

$$
\begin{aligned}
& \lim _{t \rightarrow \infty} \alpha^{-k} x^{(k)}=\bar{A}^{k} x^{(0)}=\left(p x^{(0)}\right) \bar{x}, \\
& \lim _{t \rightarrow \infty} \alpha^{-k} p^{(0)}=p^{(0)} \bar{A}^{k}=\left(p^{(0)} \bar{x}\right) p .
\end{aligned}
$$

Таким образом, предельные векторы представляют собой правый и левый фробениусовы собственные векторы, нормированные, соответственно, множителями, зависящими от начальных состояний.

В [14] показано, что в примере 3 при упоминавшемся уже условии неразложимости и примитивности подматрицы, соответствующей дугам оптимальных контуров при достаточно больших $k$, аналогично (16),

$$
A^{\otimes k}=(\bar{x}, k-1) \otimes p .
$$

Отсюда следует, что

$$
\begin{aligned}
& x^{(k)}=\left(A^{\otimes k}, k\right) \otimes x^{(0)}=(\bar{x}, k-1) \otimes(p, 1) \otimes x^{(0)}=\beta^{k-1}\left(p \otimes x^{(0)}\right) \boxplus \bar{x}, \\
& p^{(k)}=p^{(0)} \otimes A^{\otimes k}=\left(p^{(0)}, 1\right) \otimes(\bar{x}, k-1) \otimes p=\left(p^{(0)} \otimes \bar{x}\right) \beta^{(k)} \boxplus p .
\end{aligned}
$$

Здесь символ $\boxplus$ означает, что число $\beta^{k-1}\left(p \otimes x^{(0)}\right)$ в (19) и число $\left(p^{(0)} \otimes \bar{x}\right) \beta^{k}$ в (20) прибавляются ко всем элементам вектора. Эти слагаемые аналогичны нормирующим множителям в (17) и (18). Заметим также, что равенство (15) аналогично (13).

Из (19) следует, что $x^{(k)} \rightarrow \bar{x}$ при $k \rightarrow \infty$, при этом, начиная с некоторого $k$, сходимость становится монотонной: если $p \otimes x^{(0)}>0\left(p \otimes x^{(0)}<0\right)$, то $x^{(k)}>\bar{x}$ (соответственно, $x^{(k)}<\bar{x}$ ) при всех достаточно больших $k$. 


\section{Список литературы}

1. Беллман Р., Динамическое программирование. ИЛ, Москва, 1960.

2. Воробьев Н. Н., Экстремальная алгебра положительных матриц. Elektron. Inform.-verarb. Kybernetik (1967) 3, 39-71.

3. Воробьев Н. Н., Экстремальная алгебра неотрицательных матриц. Elektron. Inform.-verarb. Kybernetik (1970) 6, 303-312.

4. Романовский И. В., Оптимизация стационарного управления дискретным детерминированным процессом. Кибернетика (1967), № 2, 66-78.

5. Романовский И. В., Алгоритмы решения экстремальных задач. Наука, Москва, 1977.

6. Cuninghame-Green R., Minimax algebra. Springer, Berlin, 1979.

7. Матвеенко В. Д., Оптимальные траектории схемы динамического программирования и экстремальные степени неотрицательных матриц. Дискретная математика (1990) 2, №1, 59-71.

8. Матвеенко В. Д., О дискретных сублинейных и суперлинейных операторах. Дискретная математика (1998) 10, №2, 87-100.

9. Matveenko V. D., Development with positive externalities: the case of the Russian economy. J. Policy Modeling (1995) 17, №3, 207-221.

10. Sanchez E., Resolution of eigen fuzzy sets equations. Fuzzy Sets Syst. (1978) 1, 69-74.

11. Butkovič P., Cechlárová K., Szabó P., Strong linear independence in bottleneck algebra. Linear Algebra Appl. (1987) 94, 133-155.

12. Cechlárová K., Eigenvectors in bottleneck algebra. Linear Algebra Appl. (1992) 175, 63-73.

13. Cechlárová K., Efficient computation of the greatest eigenvector in fuzzy algebra. Tatra Mt. Math. Publ. (1997) 12, 73-79.

14. Матвеенко В. Д., Структура оптимальных траекторий дискретной детерминированной схемы с дисконтированием. Дискретная математика (1998) 10, №3, 100-114.

15. Матвеенко В. Д., Структура траекторий и вторая функция-значение в оптимизационных динамических системах с дисконтированием. Автоматика и телемеханика (1999), № 1, 26-34.

16. Вагнер Г., Основы исследования операций. Мир, Москва, 1973.

17. Дудников П. И., Самборский С. Н., Эндоморфизмы полумодуля над полукольцом с идемпотентной операцией, Препринт. ИМ АН УССР, Киев, 1987.

18. Дудников П. И., Самборский С. Н., Эндоморфизмы полумодулей над полукольцами с идемпотентной операцией. Изв. АН СССР, сер. матем. (1991) 55, №1, 93-109.

19. Маслов В. П., Колокольцев В. Н., Идемпотентный анализ и его применение в оптимальном управлении. Физматлит, Москва, 1994.

20. Zimmermann U., Linear and combinatorial optimization in ordered algebraic structures. Ann. Discrete Math. (1981) 10, 1-380.

21. Seneta E., Nonnegative matrices. An introduction to theory and applications. Allen \& Unwin, London, 1973.

22. Ашманов С. А., Введение в математическую экономику. Наука, Москва, 1984.

Статья поступила 2.06.2005. 